\title{
Natural Convection, Solute Trapping, and Channel Formation during Solidification of Saltwater
}

\author{
M. G. Worster \\ Institute of Theoretical Geophysics, Department of Applied Mathematics and Theoretical Physics, \\ Silver Street, Cambridge CB3 9EW, U.K.
}

\section{J. S. Wettlaufer*}

Applied Physics Laboratory and Department of Physics, Box 355640, University of Washington, Seattle, Washington, 98105

Received: October 11, 1996; In Final Form: March 7, $1997^{\otimes}$

\begin{abstract}
We present new experimental results relating to the growth and evolution of sea ice. These show, in particular, that brine initially remains trapped in the interstices of the sea ice, only draining into the underlying ocean once the depth of the sea-ice layer exceeds a critical value. A general theory for convection within mushy layers is applied to develop a hypothesis for when brine drainage occurs, which is tested against the experimental results.
\end{abstract}

When ice grows from water-containing impurities, it typically forms a matrix of crystals, and the impurities are rejected into the interstices of the matrix. This is the structure of sea ice, for example, which grows at the surface of the polar oceans when they are cooled from above during winter. In sea ice, the solid matrix is composed of pure ice platelets with randomly oriented horizontal $c$ axes. ${ }^{1}$ The impurities, principally salt but also man-made pollution and biological organisms, are all concentrated in the interstices between the ice platelets. These impurities increase the density of the interstitial liquid and thus provide the potential energy to drive convection. Convective exchange between the interstitial fluid and the fluid below the growing sea ice has profound influences on the structure of the ice crystals, the salt flux delivered to the polar oceans, the transport of pollutants and the evolution of algae communities. We describe here a recent experimental study of convection during the growth of sea ice ${ }^{2}$ and some of the fundamental theoretical work that can be applied to elucidate the experimental observations. These demonstrate how and why the interstitial brine initially remains confined within the sea ice and identify a critical condition for the onset of brine drainage.

The growth of sea ice is but one example of the solidification of multicomponent melts, or alloys. Such solidification has previously been studied in the context of metallurgical and geological applications. ${ }^{3}$ The two-phase region comprising essentially pure crystals of one of the components of the melt bathed in liquid enriched in the rejected components is called a mushy layer. Mushy layers owe their existence to a morphological instability of the solid-liquid interface ${ }^{4}$ and are ubiquitous during the solidification of alloys.

The physical processes involved in the evolution of sea ice can be studied in the laboratory using aqueous salt solutions. Previous laboratory studies have focused on the haline convection adjacent to the freezing front (see ref 5 and references therein), but these leave open the question of the dynamics responsible for the structure of the material. In a series of 13 experiments, ${ }^{2}$ solutions of different initial concentrations of $\mathrm{NaCl}$ (ranging from 1 to $14 \mathrm{wt} \%$ ) were placed in a rectangular cell $20 \times 20 \times 28 \mathrm{~cm}$, the upper boundary of which was cooled to

\footnotetext{
${ }^{\otimes}$ Abstract published in Advance ACS Abstracts, June 15, 1997.
}

various fixed temperatures (ranging from -10 to $-20^{\circ} \mathrm{C}$ ) below the liquidus temperature of the solution. The experimental conditions thus encompassed typical polar conditions, and the measured freezing rates were similar to those observed in the field. The depth of the mushy layer was measured with a millimeter scale, and the concentration of the liquid below the mushy layer was measured with a refractometer. Additionally, the experimental chamber was sealed, and a tube exiting the base led to a graduated buret to record the amount of expansion during solidification as a function of time. This provided the primary measurement from which the volume of ice grown was determined.

In each experiment a mushy layer of ice crystals grew downward from the cooled boundary. There was evidence from shadowgraph images of weak, distributed compositional convection once the mushy layer formed (Figure 1a). Despite this convection, the concentration of the liquid region below the mushy layer remained constant to within the resolution of the measurements (Figure 2a). After some time, however, the character of the convection changed, becoming focused into strong, isolated convective plumes (Figure 1b). We call this latter style of convection "internally driven convection". At this stage in the experiment, the concentration of the liquid region was increasing significantly as the mushy layer grew (Figure 2a), indicating a substantial flux of brine from the interior of the mushy layer. Closer inspection revealed that the convective plumes emanated from channels in the mushy layer (Figure 1c). Such brine channels have been observed in sea ice ${ }^{6,7}$ and in castings of metallic alloys, ${ }^{8}$ where they are called "chimneys". The formation of brine channels has a significant impact on the structure of the mushy layer and on the convective exchange between the mushy layer and the underlying liquid.

In particular, because the brine draining through channels in the mushy layer is replaced by fresher liquid from below, it allows further growth of ice in the layer, which increases its solid fraction (Figure 2b). The solid fraction is an important variable of sea ice, affecting its thermal, ${ }^{9}$ acoustic, ${ }^{10}$ electromagnetic, ${ }^{11}$ and mechanical ${ }^{12}$ properties. It is clear from Figure $2 b$ that the solid fraction increases rapidly once internally driven convection begins. 
(a)

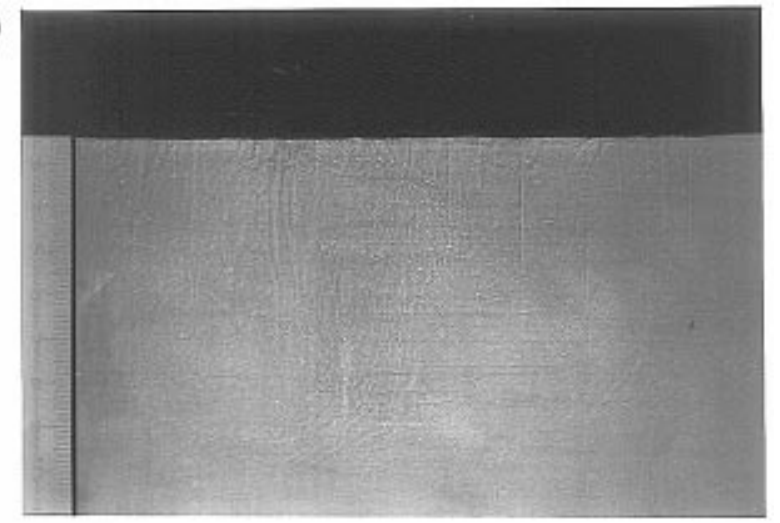

(b)

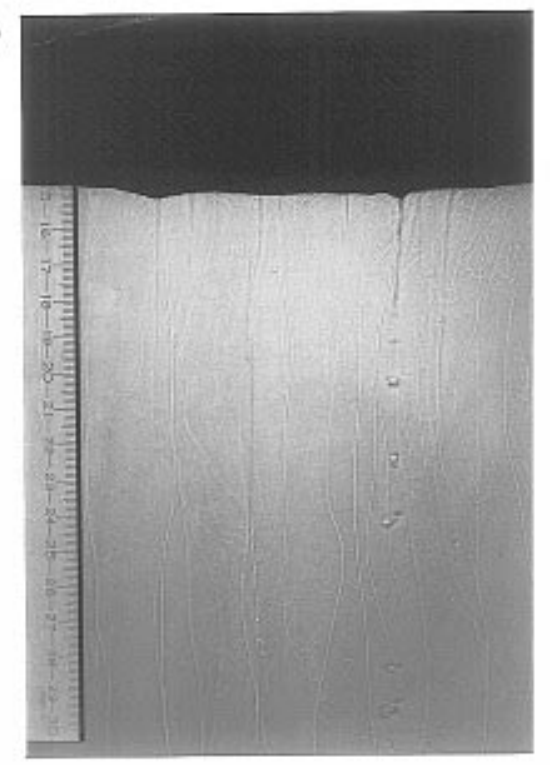

(c)

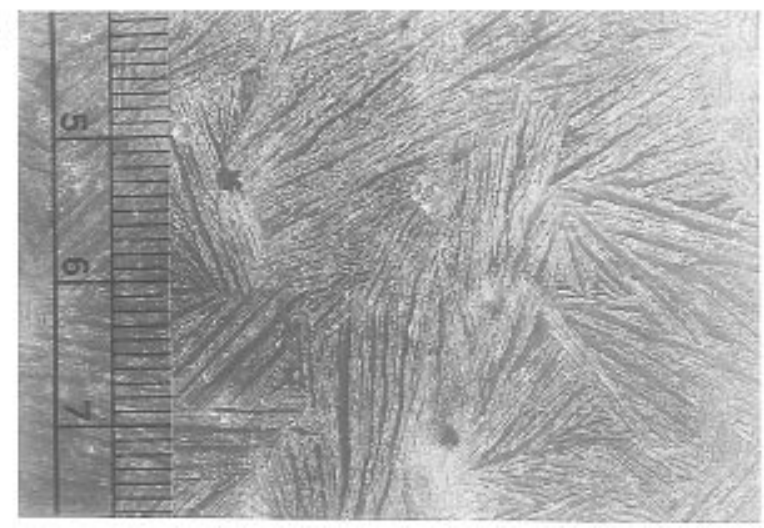

Figure 1. Photographs taken of an experiment in which an aqueous solution of $\mathrm{NaCl}$ was cooled from above. The initial concentration was 7 wt $\%$ and the cold-plate temperature was $-15^{\circ} \mathrm{C}$. (a) Early in the experiment fine-scale, distributed compositional convection was observed originating from the mush-liquid interface. (b) Several hours into the experiment we observed convection in the form of isolated plumes originating from the interior of the mushy layer. These plumes emanated from brine channels in the mushy layer, seen end-on in photograph (c). The scale on the left is in centimeters.

The two styles of compositional convection seen in the experiments can be understood in terms of a general theory of convection in mushy layers, ${ }^{13}$ which also predicts a criterion for the onset of internally driven convection. Rather than calculating the microstructural morphology of the solid phase, the theory of mushy layers provides predictions of the properties of the mushy layer averaged over the microscale. Therefore, the theory can be applied to different chemical systems

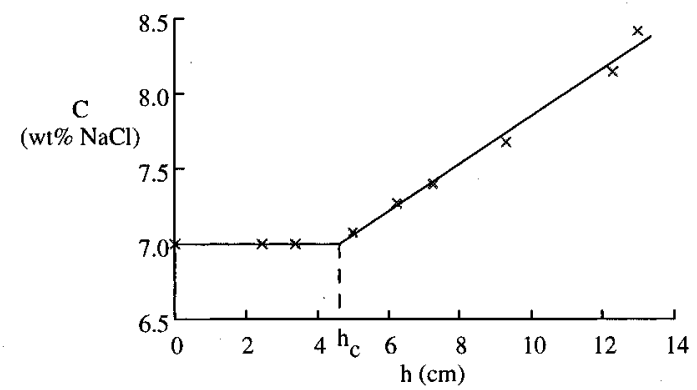

(a)

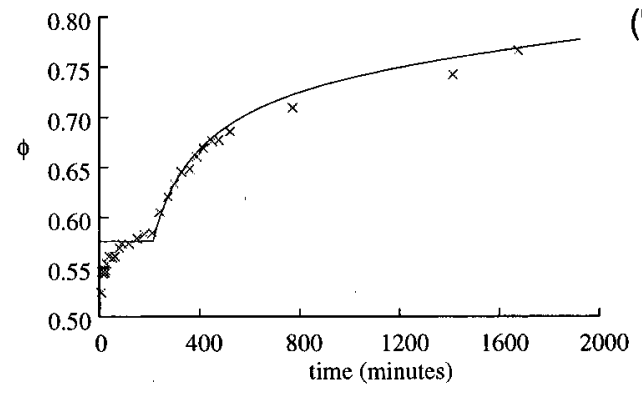

(b)

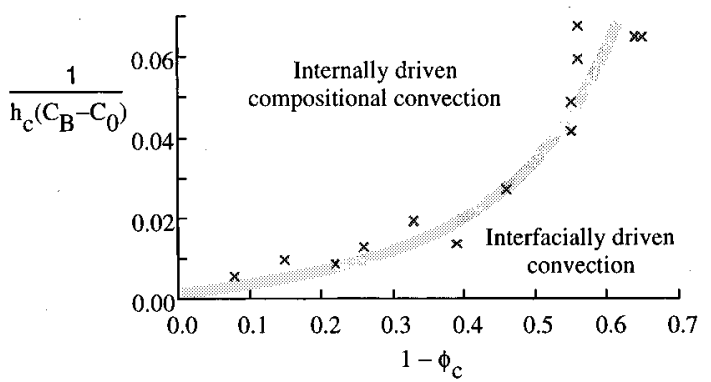

(c)

Figure 2. (a) Concentration of the liquid below the mushy layer as a function of the depth of the layer $h$. Initially, even though there is some weak compositional convection (Figure 1a), the concentration remains constant, indicating that there is little salt flux from the mushy layer. Once the depth of the mushy layer exceeds a critical value $h_{\mathrm{c}}$ the concentration of the liquid region increases with time, indicating a significant flux of brine draining from the mushy layer. (b) The draining brine is replaced by fresher water from below, which promotes additional freezing in the interior of the mushy layer causing the solid fraction $\phi$ to increase. (c) The critical point at which internally driven convection begins is determined by the magnitude of the Rayleigh number. This hypothesis (eq 13) provides a good collapse of the experimental data.

producing mushy layers of very different internal morphologies. It is striking, for example, that the circular brine channels shown in Figure 1c apparently have no regard for the platelike microstructure of the matrix of ice crystals. One might have anticipated, for example, that the convection responsible for channel formation would be so influenced by the anisotropy of the permeability of a medium comprised of platelets that channels might not form at all or that they might be more elliptical in cross section, aligned with the local platelets. Neither do the channels appear to form preferentially at grain boundaries. As we discuss below, the dominant influences in determining the formation of channels are heat and mass transport coupled with the constraint of two phase equilibrium.

The dependent variables calculated in the theory of mushy layers are the local mean temperature $T$, the local mean concentration of the interstitial liquid $C$, the local mean solid fraction $\phi$ and the local mean velocity $\mathbf{u}$. They are related by equations expressing conservation of heat,

$$
\rho C_{\mathrm{p}}\left(\frac{\partial T}{\partial t}+\mathbf{u} \cdot \nabla \mathrm{T}\right)=\nabla(k \nabla T)+L \frac{\partial \phi}{\partial t}
$$


conservation of solute,

$$
(1-\phi)\left(\frac{\partial C}{\partial t}+\mathbf{u} \cdot \nabla C\right)=\left(C-C_{\mathrm{s}}\right) \frac{\partial \phi}{\partial t}
$$

and a momentum equation,

$$
\mathbf{u}=\Pi(-\nabla p+\rho \mathbf{g})
$$

The latter is Darcy's equation, used to describe flow in a porous medium of permeability $\Pi$, which is a function of the local solid fraction $\phi$. The buoyancy term, proportional to the gravitational acceleration $\mathbf{g}$, depends on the local temperature and concentration of the interstitial liquid according to the equation of state,

$$
\rho=\rho_{0}\left[1-\alpha\left(T-T_{0}\right)+\beta\left(C-C_{0}\right)\right]
$$

where $\rho_{0}, T_{0}$, and $C_{0}$ are reference values and $\alpha$ and $\beta$ are the thermal and solutal expansion coefficients. Here $\rho, C_{p}$, and $k$ are the density, specific heat capacity, and thermal conductivity of the mushy layer, which are, in general, functions of the solid fraction. For ease of presentation, eqs $1-3$ are written for the case that these properties are the same for the liquid and solid phases. More general expressions can be found in ref 13 . Equation 1 is an advection-diffusion equation for heat, with a source term proportional to the rate of change of the solid fraction and the latent heat per unit volume $L$. This term couples the heat transfer to the solute-conservation equation, eq 2 , in which solutal diffusivity is ignored, since it is typically much smaller than the thermal diffusivity $\kappa=k / \rho C_{p}$. The solid fraction $\phi$ is determined implicitly by a constraint that the mushy layer is in local thermodynamic equilibrium, so that the temperature and concentration are coupled by the liquidus relation,

$$
T=T_{\mathrm{L}}(C)=T_{B}+\Gamma\left(C-C_{B}\right)
$$

where $T_{B}$ is the temperature of the cold surface, $C_{B}$ is the corresponding liquidus concentration, and $\Gamma$ is the slope of the liquidus. These equations, describing the internal evolution of the mushy layer, are coupled through various interfacial conditions to the usual equations for heat, solute, and momentum conservation in the fully solid and fully liquid regions bounding the mushy layer. ${ }^{14}$

By scaling lengths with the thermal diffusion length $h=\kappa / V$, times with $\kappa / V^{2}$ and velocities with $V$, where $V$ is the solidification rate, eqs $1-3$ can be written in the dimensionless form

$$
\begin{gathered}
\frac{\partial \theta}{\partial t}+\mathbf{u} \cdot \nabla \theta=\nabla^{2} \theta+S \frac{\partial \phi}{\partial t} \\
(1-\phi) \frac{\partial \theta}{\partial t}+\mathbf{u} \cdot \nabla \theta=(C-\theta) \frac{\partial \theta}{\partial t} \\
\mathbf{u}=-R a(\nabla p+\theta \mathbf{k})
\end{gathered}
$$

where

$$
\theta=\frac{T-T_{\mathrm{L}}\left(C_{0}\right)}{\Delta T}=\frac{C-C_{0}}{\Delta C}
$$

$\Delta T=\Gamma \Delta C=T_{\mathrm{L}}\left(C_{0}\right)-T_{\mathrm{E}}$ and $\mathbf{k}$ is a unit vector in the vertical direction. This identifies the important dimensionless groups: a Stefan number

$$
S=L / C_{p} \Delta T
$$

which is the ratio of the latent heat of solidification to the sensible heat required to cool the liquid; a compositional ratio

$$
\mathrm{C}=\frac{C_{\mathrm{s}}-C}{\Delta C}
$$

which gives the difference in composition between the solid and liquid phases relative to the variations in composition of the liquid; and a Rayleigh number

$$
R a=\frac{(\beta-\alpha \Gamma) \Delta C \Pi h}{\kappa v}
$$

which measures the buoyancy force relative to the viscous dissipation in the porous medium. It is the Rayleigh number that principally determines the onset of convection in the mushy layer.

Solutions of these equations show that, without convection, the diffusion-controlled density profile due to salinity has the form sketched in Figure 3a. There is a narrow compositional boundary layer in the liquid adjacent to the mushy layer in which the density is unstably stratified. A much larger unstable density difference exists across the mushy layer itself, but the liquid in the mushy layer experiences much greater resistance to flow. A linear stability analysis of this basic state ${ }^{14}$ predicts a stability boundary of the form shown in Figure $3 \mathrm{~b}$. The stability boundary has two minima, signifying that there are two possible modes of convection: one driven by the buoyancy in the compositional boundary layer; the other driven by the buoyancy internal to the mushy layer. The streamlines corresponding to these two modes of convection are presented in Figure 3c. The relative stability of the two modes varies with the parameters of the system. It has commonly been observed, however, that the boundary-layer mode is the first to become unstable.

The two modes have quite different characteristics. The boundary-layer mode has small length scales and does not penetrate far into the mushy layer. It therefore gives rise to only a very small salt flux, most of the enriched brine being confined to the interstices of the mushy layer. We interpret the convection seen in the early stages of the experiments (Figure 1a) as originating from this boundary-layer mode of instability.

By contrast, the mushy-layer mode of convection penetrates the whole of the mushy layer and is therefore associated with a much larger flux of salt. It is this mode of instability that is believed to give rise to the internally driven convection seen in the later stages of the experiments (Figure 1b). It is triggered when the Rayleigh number exceeds a critical value $R_{c}$ indicated in Figure 3b.

The mushy-layer mode of convection also results in dissolution of the solid phase in regions where the flow is directed along the temperature gradient, away from the cold boundary. This can be understood from the following argument, illustrated in Figure 3d. As mentioned previously, the interstitial liquid in a mushy layer is in local thermodynamic equilibrium. Therefore the colder liquid near the cooled boundary is also saltier. Consider a parcel of liquid (point A in Figure 3d) within the mushy layer displaced away from the cold boundary. It is colder and saltier than its new surroundings. Thermal diffusion is about 250 times faster than the diffusion rate for salt so the parcel comes into thermal equilibrium with its surroundings without significantly changing its salinity (point B in Figure 3d). The parcel of liquid is therefore oversaturated in salt and can partially dissolve the ice crystals it surrounds until complete local equilibrium is restored (point $\mathrm{C}$ in Figure 3d). 

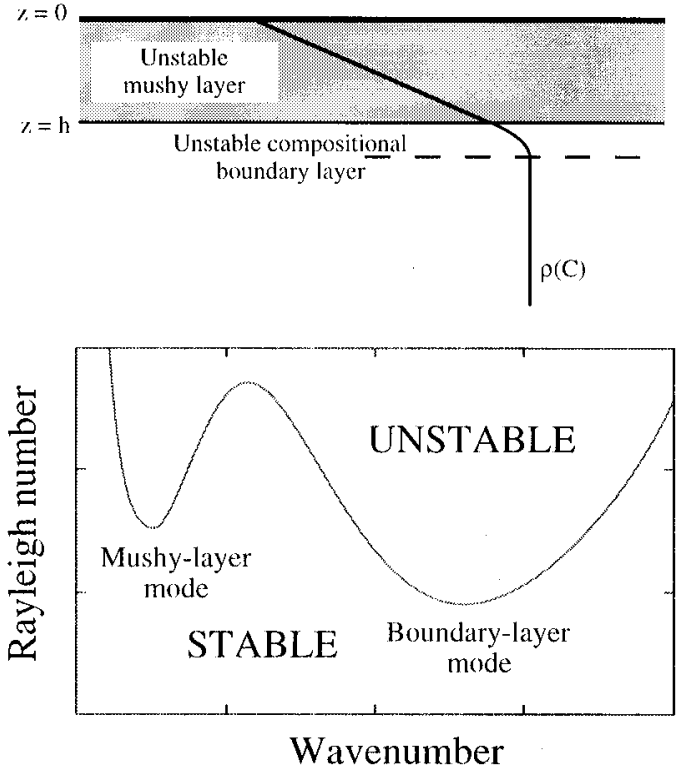

(b)

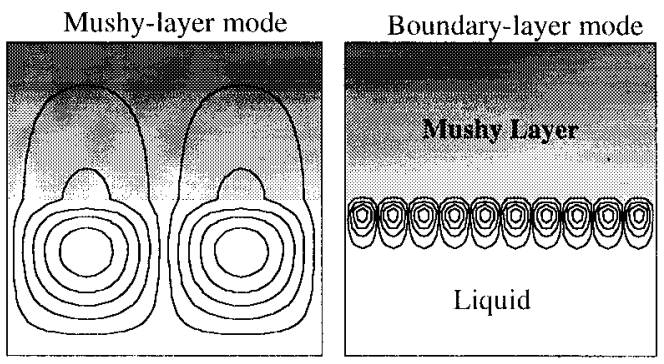

(c)

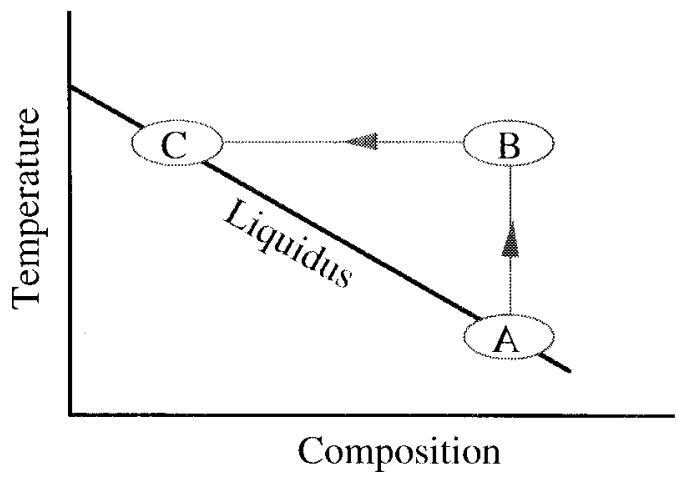

(d)

Figure 3. Diffusion-controlled density profile in the absence of convection (a) is unstably stratified throughout the mushy layer and in a narrow compositional boundary layer ahead of the mush-liquid interface. A linear stability analysis ${ }^{14}$ determines the neutral curve (b): when the Rayleigh number is larger than the values given by the neutral curve convection will occur. The two minima of the neutral curve correspond to two different modes of convection. The boundary-layer mode typically occurs first (at lower Rayleigh numbers) and gives rise to convection just in the liquid region, as shown by the streamlines (c) (cf. Figure 1a). When the mushy layer is deeper, the Rayleigh number is larger and the mushy-layer mode of convection is triggered at a value $R_{\mathrm{c}}$ corresponding to the local minimum labeled above as the "mushylayer mode". This mode penetrates the full depth of the mushy layer (c) and results in the form of convection shown in Figure 1b. (d) Sketch illustrating the evolution of a parcel of interstitial liquid displaced away from the cold boundary within the mushy layer. See text for explanation.

This mechanism of dissolution in mushy layers, by reducing the local permeability, focuses the convective flow into narrow channels, such as shown in Figure 1c. The observation of channels is further evidence that internally driven convection is associated with the mushy-layer mode of instability. We have accordingly adopted the hypothesis that internally driven convection and the concomitant formation of brine channels is initiated once the Rayleigh number exceeds a critical value. This hypothesis leads to the conclusion that

$$
\left(h_{\mathrm{c}} \Delta C\right)^{-1} \propto \Pi\left(\phi_{\mathrm{c}}\right)
$$

where $h_{\mathrm{c}}$ is the critical depth of the mushy layer, indicated in Figure $2 \mathrm{a}$, and $\phi_{\mathrm{c}}$ is the corresponding vertically averaged solid fraction.

Figure 2c shows $\left(h_{\mathrm{c}} \Delta C\right)^{-1}$ plotted as a function of the critical liquid fraction, $1-\phi_{c}$, for all 13 experiments. It can be seen that the hypothesis leads to a good collapse of the data. It is also seen that the functional form of the relationship between $\left(h_{\mathrm{c}} \Delta C\right)^{-1}$ and $1-\phi_{\mathrm{c}}$ is consistent with the expected form of the permeability function $\Pi$, the latter increasing with the liquid fraction and having positive curvature.

Figure $2 \mathrm{c}$ acts as a dynamic phase diagram for convection, showing the conditions under which brine will drain from a mushy layer. This is important for predicting the early stages of sea ice growth in leads, which are large cracks in the perennial sea-ice cover. ${ }^{15}$ The principal hypothesis underlying the study of leads is that the large scale uniformity of the Arctic mixed layer is created entirely from the brine flux of a relatively small area of leads. The geometry and forcing conditions of the experiments are directly relevant to the growth of undeformed lead ice in $^{2,15}$ and thereby provide a link between local ice properties and the brine fluxes that can be delivered to the polar oceans. The latter influence the global abyssal circulation. ${ }^{16}$ In particular, this study highlights the different modes of compositional convection that can occur within sea ice. These different modes result in dramatically different salt fluxes and therefore play a significant role in determining the boundary conditions for oceanographic models, which are currently crudely parametrized. ${ }^{17}$ For typical ice drift velocities the boundary layer adjacent to the underside of sea ice where shear is negligible can be tens of centimeters or greater, and hence the convective dynamics we study are spatially unaffected by larger scale turbulence. At very large drift velocities these conditions do not prevail.

Finally, we note that haline convection exerts a controlling influence on experimental estimates of phase segregation coefficients in electrolyte solutions. ${ }^{18}$ The dynamic interactions between convection and solidification, such as those epitomized here, must be taken into account if accurate determinations are to be made.

Acknowledgment. This work has been generously supported by grants from the Office of Naval Research, the National Science Foundation, the National Aeronautics and Space Administration, and the Natural Environment Research Council of the UK. We thank our colleagues and collaborators, especially J. G. Dash and H. E. Huppert, for many stimulating conversations.

\section{References and Notes}

(1) Weeks, W. F.; Wettlaufer, J. S. In The Johannes Weertman's Symposium; Arsenault, R. J., et al., Eds.; The Minerals, Metals \& Materials Society: 1996; p 337.

(2) Wettlaufer, J. S.; Worster, M. G.; Huppert, H. E. J. Fluid Mech. 1997, 344, 291.

(3) Davis, S. H., Huppert, H. E., Müller, U., Worster, M. G., Eds. Interactive Dynamics of Convection and Solidification; NATO ASI 1992, E219. Kluwer. 
(4) Mullins, W. W.; Sekerka, R. F. J. Appl. Phys. 1964, 35, 444

(5) Wakatsuchi, M.; Ono, N. J. Geophys. Res. 1983, 88, 2943.

(6) Bennington, K. O. Some crystal growth features of sea ice. $J$. Glaciol 1963, 4, 669.

(7) Lake, R. A.; Lewis, E. L. J. Geophys. Res. 1970, 75, 583.

(8) Hellawell, A.; Sarazin, J. R.; Steube, R. S. Phil. Trans. R. Soc. London 1993, A345, 507.

(9) Schwerdtfeger, P. J. Glaciol 1963, 4, 789.

(10) Williams, K. L.; Garrison, G. R.; Mourad, P. D. J. Acoust. Soc. Am. 1992, 92, 2075.

(11) Winebrenner, D. P.; Bredow, J.; Fung, A. K.; et al. In Microwave Remote Sensing of Sea Ice; Carsey, F., Ed.; Geophysical Monograph 68; American Geophysical Union: 1992; p 137.
(12) Weeks, W. F. In IAPSO Advanced Study Institute-Summer School: Physics of Ice-Covered Seas, Leppäranta, M., Ed.; University of Helsinki: 1997.

(13) Worster, M. G. In ref 3, p 113.

(14) Worster, M. G. J. Fluid Mech. 1992, 237, 649. 393.

(15) Morison, J.; et al. The Leadex Group, Eos, Trans. AGU 1993, 74,

(16) Aagaard, K.; Carmack, E. In The Polar Regions and Their Role in Shaping the Global Environment; Johannessen, O. M., Muench, R. D. Overland, J. E., Eds.; Geophysical Monograph; American Geophysical Union: 1994; Vol. 85, p 5.

(17) Smith IV, D. C.; Morison, J. H. J. Geophys. Res. 1993, 98, 10069

(18) Gross, G. W.; Wong, P. M.; Humes, K. J. Chem. Phys. 1977, 67, 5264. 